\title{
PROPRIEDADES QUÍMICAS DE EXTRATOS TÂNICOS DA CASCA DE Pinus oocarpa E AVALIAÇÃO DE SEU EMPREGO COMO ADESIVO
}

\author{
Michel Cardoso Vieira ${ }^{1}$, Roberto Carlos Costa Lelis ${ }^{1}$, Nayara Dorigon Rodrigues ${ }^{1}$
}

*Autor para correspondência: mceflorestal@ig.com.br

RESUMO: Neste trabalho, objetivou-se avaliar as propriedades de extratos aquosos obtidos da casca de Pinus oocarpa sob adição de sulfito de sódio e bissulfito de sódio e a possibilidade de emprego dos taninos da casca como adesivo para colagem de madeira. Para a casca de Pinus oocarpa, foram testadas oito formas de extração dos taninos. Após a avaliação das propriedades químicas dos extratos tânicos, optou-se por empregar a extração com água destilada sob adição de $5 \%$ de sulfito de sódio para confecção do adesivo tanino-formaldeído. Adesivo Fenol-formaldeído e Uréia-formaldeído foram substituídos por 10\% de tanino de Pinus oocarpa e o efeito dessa adição sobre a qualidade do adesivo foi avaliado. A adição de extrato tânico da casca de Pinus oocarpa mostrou que é possível utilizar tanino puro como adesivo, em razão de suas boas características de colagem. A adição do extrato tânico aos adesivos sintéticos contribuiu para o aumento nos valores de viscosidade. Dessa forma, a substituição de adesivos sintéticos por taninos é possível somente até $10 \%$.

Palavras-chave: Extrativo, polifenóis, tanino.

\section{CHEMICAL PROPERTIES OF TANNIC EXTRACTS FROM BARK OF Pinus oocarpa AND THEIR USE AS ADHESIVE}

\begin{abstract}
This work evaluated the properties of aqueous extracts obtained from the bark of Pinus oocarpa under addition of sodium sulfite and sodium bisulfite and the possibility of employment of tannins from the bark as an adhesive for bonding wood. After evaluation of the chemical properties of tannic extracts it was decided to employ the extraction with distilled water under addition of $/ 5 \%$ sodium sulfite to prepare for the tannin-formaldehyde adhesive. Adhesive phenol formaldehyde and urea-formaldehyde were modified with 10\% tannin Pinus oocarpa and the effect of this addition on the quality of the adhesive was evaluated. The addition from the bark of Pinus oocarpa showed that it is possible to use pure tannin as an adhesive because of its good gluing characteristics. The addition of tannic extract to synthetic adhesives contributed to increase viscosity values. Thus the substitution of synthetic adhesives by tannins is possible only up to $10 \%$.
\end{abstract}

Key words: Extract, polyphenol, tannin.

\section{INTRODUÇÃO}

Os taninos são produtos naturais presentes em várias fontes renováveis, como por exemplo, na casca de acácia negra (Acacia mearnsii), Pinus radiata e na madeira do cerne de quebracho (Schinopsis sp) (MORI et al., 2001). Lopes et al. (2003) destacam o estudo sobre o teor de taninos nas diferentes espécies vegetais, pelas aplicações industriais no curtimento de couro, na fabricação de adesivos e às propriedades farmacológicas.

Os taninos são considerados polifenóis, em razão da grande quantidade de anéis fenólicos presentes em sua estrutura, com massa molecular variando entre 500 a 3000 $\mathrm{g} / \mathrm{mol}$ aproximadamente. Os taninos são classificados quimicamente em taninos hidrolisáveis e taninos condensados. Os taninos hidrolisáveis apresentam na sua constituição monômeros de ácido gálico ou ácido elágico, enquanto que os taninos condensados são polifenóis com peso molecular variado, consistindo de unidades flavonóidicas (flavan-3-ol e flavan-3,4-diol), em vários graus de condensação (HOINACKI et al., 1994; MORI et al., 2003).

Normalmente, os taninos são obtidos pela extração da casca ou madeira em água, podendo-se adicionar sais inorgânicos, como sulfito de sódio $\left(\mathrm{Na}_{2} \mathrm{SO}_{3}\right)$, carbonato de sódio $\left(\mathrm{Na}_{2} \mathrm{CO}_{3}\right)$ e bissulfito de sódio $\left(\mathrm{NaHSO}_{3}\right)$ (PIZZI, 1983). A extração aquosa de taninos condensados na presença de sulfito de sódio é chamada sulfitação, sendo especialmente adequada para a produção de extratos tânicos destinados à síntese de adesivos. Com a sulfitação, podem ser obtidos taninos mais solúveis em água e extratos com viscosidade mais baixa. Maiores informações sobre o processo de sulfitação podem ser obtidas em Pizzi (1983) e Silva (2001).

$\mathrm{Na}$ indústria de painéis de madeira, são empregados principalmente os adesivos sintéticos Ureia-formaldeído (UF) e Fenol-formaldeído (FF). No Brasil, trabalhos envolvendo taninos como adesivo para madeira foram

${ }^{1}$ Universidade Federal Rural do Rio de Janeiro - Seropédica, Rio de Janeiro, Brasil

Cerne, Lavras, v. 20, n. 1, p. 47-54, jan./mar. 2014 
iniciados no final da década de setenta e início de oitenta (COPPENS et al., 1980; SANTANA; PASTORE JUNIOR, 1981). Em razão da oscilação no preço do petróleo e à fragilidade dos produtos sintéticos advindos de fontes de matérias-primas não-renováveis, a utilização de resinas à base de extratos de casca de espécies florestais tem sido alvo de inúmeras investigações (CARNEIRO et al., 2001, 2009; HILLIG et al., 2002; HOONG et al., 2011; MELO et al., 2010; MORI et al., 1999; PAES et al., 2006; TOSTES et al., 2004; VALENZUELA et al., 2012).

No Brasil, a área total reflorestada com Pinus spp. é de 1,8 milhões de hectares, sendo a madeira empregada nos segmentos como celulose e papel, móveis e madeira processada mecanicamente, que engloba a produção de madeira serrada, painéis reconstituídos, compensados e laminados. A produção de madeira em toras de pinus concentra-se nas regiões Sudeste e Sul, as quais correspondem a $95,3 \%$, com 46,5 milhões de $\mathrm{m}^{3} /$ ano da produção sustentável nacional.

Dessa forma, considerando-se o volume de madeira em tora produzida e que várias toneladas de cascas são produzidas anualmente, um possível uso alternativo para essas cascas seria a extração de taninos para a produção de adesivos para madeira, sendo que o resíduo obtido após a extração poderia ser queimado para geração de energia.

Neste trabalho, objetivou-se avaliar as propriedades químicas de extratos tânicos obtidos da casca de Pinus oocarpa sob adição de sulfito de sódio e bissulfito de sódio, bem como a viabilidade de emprego de taninos da casca como adesivos e em misturas com adesivos sintéticos.

\section{MATERIAL E MÉTODOS}

\subsection{Obtenção do material}

As cascas foram coletadas em povoamentos de Pinus oocarpa, da empresa Duratex, em Agudos-SP. Cinco árvores com DAP variando de 15 a $40 \mathrm{~cm}$ tiveram suas cascas removidas com auxílio de um facão. $\mathrm{O}$ material foi seco ao ar, sendo fragmentado em moinho de martelo, peneirado e armazenado. Esse material foi utilizado nas extrações para obtenção dos taninos.

\subsection{Extração da casca}

A casca foi extraída com água sob adição de bisulfito de sódio $\left(\mathrm{NaHSO}_{3}\right)$ e sulfito de sódio $\left(\mathrm{Na}_{2} \mathrm{SO}_{3}\right)$ em diferentes concentrações originando 8 tratamentos. O material foi extraído em balão sob refluxo por 2 horas utilizando-se uma relação casca/licor igual a 1:15 $(\mathrm{p} / \mathrm{v})$. Após a extração, o material foi filtrado a vácuo utilizando-se cadinho de vidro sinterizado número 3. Para cada tratamento, foram realizadas cinco repetições. Os tratamentos estão esquematizados na Tabela 1.

Tabela 1 - Sais utilizados para extração de taninos da casca de Pinus oocarpa.

Table 1 - Salts used for extraction of tannin from the bark of Pinus oocarpa.

\begin{tabular}{cc}
\hline Tratamentos & Extrações* \\
\hline T1 & $\mathrm{H}_{2} \mathrm{O}$ \\
T2 & $\mathrm{H}_{2} \mathrm{O}+1 \% \mathrm{NaHSO}_{3}$ \\
T3 & $\mathrm{H}_{2} \mathrm{O}+5 \% \mathrm{NaHSO}_{3}$ \\
T4 & $\mathrm{H}_{2} \mathrm{O}+8 \% \mathrm{NaHSO}_{3}$ \\
T5 & $\mathrm{H}_{2} \mathrm{O}+10 \% \mathrm{NaHSO}_{3}$ \\
T6 & $\mathrm{H}_{2} \mathrm{O}+1 \% \mathrm{Na}_{2} \mathrm{SO}_{3}$ \\
T7 & $\mathrm{H}_{2} \mathrm{O}+5 \% \mathrm{Na}_{2} \mathrm{SO}_{3}$ \\
T8 & $\mathrm{H}_{2} \mathrm{O}+8 \% \mathrm{Na}_{2} \mathrm{SO}_{3}$ \\
\hline
\end{tabular}

* = A percentagem de sal foi calculada com base na massa seca de cascas.

\subsection{Avaliação das propriedades dos extratos}

Para cada tratamento, foram determinados os teores de extrativos e suas propriedades, a saber: teor de polifenóis condensados pelo método de Stiasny (Número de Stiasny - NS), a reatividade pelo método UV (ROFFAEL, 1976), teor de taninos, teor de não-taninos e valor do $\mathrm{pH}$, sendo realizadas cinco repetições por tratamento.

Após cada extração, foi separada uma alíquota de $25 \mathrm{ml}$ do filtrado para determinação do teor de extrativos, conforme Ferreira et al. (2009). Os teores de polifenóis condensados foram calculados pela reação de Stiasny (WISSING, 1955) e a reatividade dos mesmos pelo método Ultravioleta (UV) (ROFFAEL, 1976). Os teores de taninos e de não-taninos foram calculados através das fórmulas abaixo:

$\mathrm{TAN}=\mathrm{NS} \times \mathrm{EXT}$

$\mathrm{NTAN}=\mathrm{EXT}-\mathrm{TAN}$

Sendo: TAN $=$ Teor de taninos $(\%)$; NS = Número de Stiasny; EXT = teor de extrativos; NTAN = Teor de Nãotaninos (\%).

$\mathrm{O} \mathrm{pH}$ das soluções foi determinado por $\mathrm{pH}$-metro digital, da marca QUIMIS aparelhos científicos LTDA, à

Cerne, Lavras, v. 20, n. 1, p. 47-54, jan./mar. 2014 
temperatura ambiente, sendo o valor registrado após quatro minutos de contato do eletrodo com a solução.

\subsection{Extração em autoclave}

O tratamento que apresentou melhor rendimento em taninos foi utilizado para extração de grandes quantidades em autoclave.

A casca foi extraída em autoclave, com capacidade volumétrica de 15 litros, por um período de 2 horas, numa relação casca:licor igual a 1:15 (p/v). Após cada extração, o material foi filtrado em cadinho de vidro sinterizado número 3, colocado em bandejas de vidro que foram levadas à estufa a $103^{\circ} \pm 2^{\circ} \mathrm{C}$ por 8 horas, para uma secagem prévia do extrato. Posteriormente, o material foi removido para uma estufa a $60^{\circ} \mathrm{C}$ até a secagem completa, quando então o material foi moído, obtendo-se o extrato na forma de pó.

\subsection{Propriedades dos extratos tânicos da casca de Pinus oocarpa}

Para a determinação das propriedades dos extratos tânicos foi confeccionada uma solução de extrato tânico a $45 \%$. As seguintes propriedades foram avaliadas: viscosidade, teor de sólidos, tempo de formação de gel e pH. Para cada análise, foram realizadas cinco repetições, procedendo-se, posteriormente, à análise de variância e aplicação do teste de Scott-knott, quando necessário.

Após preparo e homogeneização da solução de tanino a $45 \%$, a viscosidade foi determinada, utilizandose o copo Ford (AMERICAN SOCIETY FOR TESTING AND MATERIALS - ASTM, 1994). O teor de sólidos foi determinado segundo Tostes et al. (2004). O tempo de formação de gel foi determinado conforme descrito por Tostes et al. (2004), utilizando-se $10 \mathrm{~g}$ da solução de extrato tânico a $45 \%$ e solução de formaldeído a $37 \%$ (catalisador) na proporção de $20 \%$ sobre o teor de sólidos contidos na solução do extrato.O pH da solução de extrato tânico a $45 \%$ foi determinado por pH-metro digital, da marca QUIMIS Aparelhos Científicos LTDA, à temperatura ambiente. $\mathrm{O}$ valor do $\mathrm{pH}$ foi registrado após 4 minutos de contato do eletrodo com a solução. Foram feitas cinco repetições por tratamento.

2.6 Propriedades dos adesivos Uréia-formaldeído (UF), Fenol-formaldeído (FF) e de suas modificações com extrato tânico da casca de Pinus oocarpa
As propriedades dos adesivos sintéticos MDP 9020 (UF) e HL 7090 (FF) e dos adesivos modificados com tanino da casca de Pinus oocarpa foram avaliadas. As modificações dos adesivos sintéticos foram feitas pela substituição de parte dos adesivos UF e FF por uma solução de extrato tânico a 45\% na razão de 90:10, 80:20 e 70:30. Para cada adesivo, foram determinadas as seguintes propriedades: viscosidade, teor de sólidos, tempo de formação de gel e $\mathrm{pH}$. As determinações foram feitas utilizando-se cinco repetições, seguindo-se as metodologias descritas no item 2.5.

O tempo de formação de gel foi determinado com as seguintes alterações: Para o adesivo UF e de suas modificações foi utilizado como catalisador uma solução de sulfato de amônia a $24 \%$ na razão de $2 \%$ sobre a massa total de sólidos. No caso do adesivo FF e de suas modificações, foi utilizado como catalisador uma solução de carbonato de potássio a $60 \%$ na razão de $6 \%$ sobre a massa total de sólidos.

\section{RESULTADOS E DISCUSSÃO}

3.1 Avaliação dos teores de extrativos, das propriedades químicas, dos teores de taninos e de não-taninos da casca de Pinus oocarpa

Na Tabela 2, são mostrados os valores médios dos teores de extrativos, das propriedades químicas, dos teores de taninos e de não-taninos da casca de Pinus oocarpa, extraídas com água e com água sob adição de diferentes percentuais de bisulfito de sódio $\left(\mathrm{NaHSO}_{3}\right)$ e sulfito de sódio $\left(\mathrm{Na}_{2} \mathrm{SO}_{3}\right)$.

O rendimento em extrativos é um parâmetro inicial na caracterização das substâncias encontradas na casca, pois, por meio deste pode-se definir qual tratamento terá maior produtividade e a forma mais efetiva de obtenção dos extrativos.

A adição dos diferentes sais acarretou diferentes valores de percentagem de extrativos. Para bisulfito, a adição de $5 \%$ e $8 \%$ favoreceu para maiores valores de extrativos quando comparados com água pura; entretanto, a diferença não foi significativa. Para sulfito de sódio, somente a adição de $5 \%$ de sulfito foi estatisticamente superior quando comparado com água pura, sendo inclusive superior a todos os tratamentos com bisulfito. Ferreira et al. (2009) encontraram teores de extrativos com a adição de 5\% de sulfito de sódio de $32,49 \%$ na casca de Pinus oocarpa, sendo os valores próximos ao encontrado neste trabalho.

Cerne, Lavras, v. 20, n. 1, p. 47-54, jan./mar. 2014 
Tabela 2 - Valores médios e comparação das médias para os teores de extrativos, teores de polifenóis (NS), \% de taninos; de nãotaninos, $\mathrm{pH}$ e reatividade nos diferentes tratamentos com a casca de Pinus oocarpa.

Table 2 - Mean values and comparison of means for the extract content, the polyphenols (NS), \% tannin, no tannins, pH and reactivity in the different treatments from the bark of Pinus oocarpa.

\begin{tabular}{lcccccc}
\hline Tratamento & \% Extrativos & NS & $\%$ Tanino & \% Não- tanino & pH & Reatividade (UV) \\
\hline T1 $\left(\mathrm{H}_{2} \mathrm{O}\right)$ & $24,2 \mathrm{~b}$ & $61,6 \mathrm{c}$ & $15,06 \mathrm{c}$ & $9,18 \mathrm{a}$ & $3,14 \mathrm{e}$ & $83,30 \mathrm{~b}$ \\
$\mathrm{~T} 2\left(\mathrm{H}_{2} \mathrm{O}+1 \%\right.$ bisulfito) & $18,9 \mathrm{c}$ & $87,3 \mathrm{a}$ & $16,46 \mathrm{c}$ & $2,40 \mathrm{c}$ & $3,22 \mathrm{e}$ & $98,30 \mathrm{a}$ \\
$\mathrm{T} 3\left(\mathrm{H}_{2} \mathrm{O}+5 \%\right.$ bisulfito) & $25,9 \mathrm{~b}$ & $71,1 \mathrm{~b}$ & $18,38 \mathrm{~b}$ & $7,52 \mathrm{~b}$ & $3,20 \mathrm{e}$ & $99,01 \mathrm{a}$ \\
$\mathrm{T} 4\left(\mathrm{H}_{2} \mathrm{O}+8 \%\right.$ bisulfito) & $26,1 \mathrm{~b}$ & $72,9 \mathrm{~b}$ & $19,06 \mathrm{~b}$ & $7,06 \mathrm{~b}$ & $3,57 \mathrm{~d}$ & $99,39 \mathrm{a}$ \\
$\mathrm{T} 5\left(\mathrm{H}_{2} \mathrm{O}+10 \%\right.$ bisulfito $)$ & $23,2 \mathrm{~b}$ & $85,1 \mathrm{a}$ & $19,66 \mathrm{~b}$ & $3,62 \mathrm{c}$ & $3,64 \mathrm{~d}$ & $98,09 \mathrm{a}$ \\
$\mathrm{T} 6\left(\mathrm{H}_{2} \mathrm{O}+1 \%\right.$ sulfito $)$ & $16,8 \mathrm{c}$ & $88,8 \mathrm{a}$ & $14,94 \mathrm{c}$ & $1,86 \mathrm{c}$ & $4,87 \mathrm{c}$ & $99,42 \mathrm{a}$ \\
$\mathrm{T} 7\left(\mathrm{H}_{2} \mathrm{O}+3 \%\right.$ sulfito $)$ & $25,4 \mathrm{~b}$ & $87,8 \mathrm{a}$ & $20,40 \mathrm{~b}$ & $2,85 \mathrm{c}$ & $5,20 \mathrm{~b}$ & $99,50 \mathrm{a}$ \\
$\mathrm{T} 8\left(\mathrm{H}_{2} \mathrm{O}+5 \%\right.$ sulfito $)$ & $30,1 \mathrm{a}$ & $89,9 \mathrm{a}$ & $27,09 \mathrm{a}$ & $3,05 \mathrm{c}$ & $5,68 \mathrm{a}$ & $99,53 \mathrm{a}$ \\
\hline
\end{tabular}

Letras iguais, dentro de uma mesma coluna, não diferem estatisticamente entre si, ao nível de 95\% de significância, pelo teste de Scott-Knott.

O Número de Stiasny (NS) representa o percentual de taninos condensados existentes no extrato, pela reação com formaldeído e ácido clorídrico (reação de stiasny. Os resultados obtidos para a casca de Pinus oocarpa monstraram que a adição de sais favoreceu a solubilização dos polifenóis, havendo diferença significativa entre todos os tratamentos, quando comparados com o obtido somente com água. A extração na concentração de 1\%, para sulfito e bisulfito, não ocasionou alterações nos índices de Stiasny (NS) dos extratos obtidos. Para 5\% de sulfito e $5 \%$ bisulfito (T8 e T3), os maiores valores de NS foram obtidos nas extrações com adição de sulfito, sendo os mesmos significativos. De modo geral, os maiores valores de NS foram obtidos nas extrações com adição de sulfito de sódio.

Ferreira et al. (2009) encontraram Índice de Stiasny (NS) de $97,32 \%$ com a adição de $5 \%$ de sulfito de sódio na casca de Pinus oocarpa, sendo os valores médios mais elevados aos encontrados neste trabalho.

Com relação à percentagem de taninos, nota-se que a adição de bisulfito de sódio ao processo de extração elevou o rendimento em taninos dos extratos quando comparados aos obtidos apenas com água destilada, sendo que houve diferença significativa somente nos tratamentos que utilizaram maiores concentrações desse sal (T3, T4 e T5). Na extração, utilizando-se sulfito de sódio, o aumento da concentração do sal ocasionou a obtenção de maiores percentuais de taninos (T7 e T8), sendo os maiores valores de taninos obtidos com adição de 5\% de sulfito de sódio. Comparando-se os dois sais nas diferentes concentrações, os maiores teores de taninos foram obtidos para sulfito na concentração de $5 \%$, sendo significativo com todos os outros tratamentos.

Ferreira et al. (2009) encontraram valores de taninos na casca de Pinus oocarpa em extração com água destilada sob adição de $5 \%$ de sulfito de sódio de $31,63 \%$, sendo os valores próximos ao encontrado neste trabalho.

De modo geral, os menores valores de não-taninos foram encontrados nas extrações com água sob adição de sulfito de sódio, sendo os valores significativos quando comparados aos valores médios encontrados na extração com água (T1). Os maiores valores de não-taninos foram encontrados no tratamento em que foi utilizada somente água na extração (T1). Os não-taninos correspondem à fração de açúcares e outros extrativos não fenólicos presentes nos extratos da casca, sendo que os mesmos podem influenciar negativamente a qualidade do adesivo, uma vez que contribuem para o aumento da viscosidade dos adesivos, em razão do alto peso molecular.

Os valores encontrados para não-taninos foram superiores aos encontrados por Ferreira et al. (2009) na casca de Pinus oocarpa, que em extração com água destilada e com água sob adição de 5\% de sulfito de sódio, encontraram valores de $2,10 \%$ e $0,86 \%$, respectivamente.

$\mathrm{O}$ valor de $\mathrm{pH}$ é de grande importância na reatividade dos taninos, pois a acidez interfere na reação de condensação do tanino com o formaldeído. De modo geral, nota-se que a adição dos sais aumentou o valor $\mathrm{pH}$ dos extratos. Esse aumento foi significativo para todas as concentrações de sulfito de sódio. Os extratos obtidos no tratamento com água pura apresentaram os menores

Cerne, Lavras, v. 20, n. 1, p. 47-54, jan./mar. 2014 
valores de $\mathrm{pH}$, distinguindo-se o caráter ácido dos taninos em extratos aquosos.

Ferreira et al. (2009) encontraram valores de pH na casca de Pinus oocarpa em extração com água destilada e com a adição de 5\% de sulfito de sódio de 3,35 e 5,28 respectivamente, sendo próximos dos resultados encontrados neste trabalho.

A quantificação dos polifenóis, por meio da absorção ultravioleta (UV) é considerada mais eficaz que o método de Stiasny, pois considera também os polifenóis que reagem com o formaldeído sem se precipitarem. Por meio dessa metodologia pode-se observar que os valores médios encontrados para a reatividade foram elevados, evidenciando a grande reatividade dos polifenóis (taninos) da casca de Pinus oocarpa. Estatisticamente, houve diferença significativa apenas entre os resultados encontrados nas extrações com água destilada e as demais formas de extração.

Teodoro (2008) encontrou valores de reatividade na casca de Eucalyptus pellita em extração com $2 \%$ de $\mathrm{Na}_{2} \mathrm{SO}_{3}$ de $75,38 \%$, sendo abaixo dos encontrados neste trabalho. Ferreira et al. (2009) encontraram valores de reatividade na casca de Pinus oocarpa em extração com a adição de $5 \%$ de sulfito de sódio de $99,07 \%$, sendo próximos dos resultados encontrados neste trabalho.

\subsection{Avaliação das propriedades dos extratos tânicos da casca de Pinus oocarpa, dos adesivos sintéticos e de} suas modificações

Na avaliação das propriedades dos extratos tânicos da casca, foram empregados extratos obtidos em autoclave (ver ítem 2.4), utilizando-se água e sulfito de sódio a 5\%. A quantidade de sulfito foi calculada com base na massa seca de cascas. Esse tratamento foi o que apresentou melhor rendimento em taninos.

Inicialmente, foi previsto substituir $20 \%$ e 30 $\%$ do adesivo UF e FF por tanino da casca de Pinus oocarpa, mas isso não foi possível, uma vez que nestas percentagens houve um grande aumento da viscosidade, sendo impossível a sua determinação no copo Ford. Normalmente, os valores de viscosidade de soluções de tanino são muito elevados. Isso se deve, provavelmente, aos fenóis das moléculas de tanino que apresentam alto peso molecular e também aos taninos condensados reativos que formam agregados moleculares, por meio de pontes de hidrogênio, contribuindo para aumento da viscosidade.

$\mathrm{Na}$ Tabela 3, são mostrados os valores médios para teor de sólidos (TS), viscosidade (VISC), tempo
Tabela 3 - Valores médios para teor de sólidos (TS), viscosidade (VISC), tempo de formação de gel (TFG) e pH dos extratos tânicos da casca de Pinus oocarpa, dos adesivos FF e UF e suas modificações.

Table 3 - Mean values for solids (TS), viscosity (VISC), gel formation time (TFG) and $\mathrm{pH}$ of extracts tannin from the bark of Pinus oocarpa, the FF and UF adhesives and its modifications.

\begin{tabular}{lcccc}
\hline Tratamento & VISC (cP) & TS (\%) & TFG (min) & pH \\
\hline Tanino de & $1035,70 \mathrm{a}$ & $47,80 \mathrm{e}$ & $0,94 \mathrm{c}$ & $6,54 \mathrm{e}$ \\
Pinus oocarpa & & & & \\
Adesivo FF & $776,60 \mathrm{c}$ & $58,86 \mathrm{c}$ & $44,0 \mathrm{a}$ & $12,75 \mathrm{a}$ \\
$\begin{array}{l}\text { Adesivo FF + } \\
10 \% \text { Tanino }\end{array}$ & $818,60 \mathrm{~b}$ & $55,50 \mathrm{~d}$ & $37,5 \mathrm{~b}$ & $12,39 \mathrm{~b}$ \\
$\begin{array}{l}\text { Adesivo UF } \\
\text { Adesivo UF + }\end{array}$ & $524,45 \mathrm{~d}$ & $76,93 \mathrm{a}$ & $2,06 \mathrm{c}$ & $8,03 \mathrm{c}$ \\
$10 \%$ Tanino & $543,35 \mathrm{~d}$ & $65,75 \mathrm{~b}$ & $2,09 \mathrm{c}$ & $7,27 \mathrm{~d}$ \\
\hline
\end{tabular}

Médias seguidas pela mesma letra, dentro de uma mesma coluna, não diferem estatisticamente entre si ao nível de 5\% de significância pelo teste de Scott-knott.

de formação de gel (TFG) e pH dos extratos tânicos da casca de Pinus oocarpa, dos adesivos sintéticos e de suas modificações.

A adição de extrato tânico da casca de Pinus oocarpa aos adesivos Uréia- formaldeído (UF) e Fenol-formaldeído (FF) acarretou aumento dos valores de viscosidade nos adesivos, embora tenha sido significativo somente a adição de tanino ao adesivo Fenol-formaldeído (FF). Isso pode ser atribuído às associações intermoleculares das moléculas de taninos que promovem o aumento da viscosidade. Também os extrativos não fenólicos presentes no extrato tânico, de alto peso molecular, podem ter contribuído para o aumento da viscosidade (PIZZI, 1980).

Segundo Pizzi e Mittal (1994), a alta viscosidade dos extratos tânicos se deve as ligações de hidrogênio e atração eletrostática entre tanino-tanino, tanino-goma e interações goma-goma. A solução aquosa de extrato tânico não é uma solução verdadeira, mas de fato, uma suspensão coloidal na qual o acesso da água em todas as partes é baixo. Como consequência, é difícil eliminar as ligações de hidrogênio intermoleculares pela simples diluição em água.

Gonçalves e Lelis (2009), estudando tanino de acácia negra como elemento modificador da resina UF, encontraram resultados diferentes, pois a adição do extrato tânico reduziu o valor da viscosidade da substituição a

Cerne, Lavras, v. 20, n. 1, p. 47-54, jan./mar. 2014 
10\%. Gonçalves (2000) e Tostes et al. (2004) também observaram o mesmo comportamento na substituição a $10 \%$. Aqui é importante ressaltar que a viscosidade da solução de taninos pode ser também influenciada pelo valor $\mathrm{pH}$ da solução tânica. Para taninos de acácia negra, valores de $\mathrm{pH}$ acima de 9 contribuem para um aumento nos valores de viscosidade e abaixo de 7 a uma diminuição da viscosidade. Deve-se lembrar também que o tanino de acácia negra é diferente do tanino de pinus, ou seja as unidades flavonóides que compõem as moléculas de taninos são diferentes para os dois tipos de taninos. Isso pode explicar também a diferença nos resultados encontrados nesse trabalho para as modificações do adesivo UF e tanino de pinus, quando comparados aos trabalhos de Gonçalves (2000), Gonçalves e Lelis (2009) e Tostes et al. (2004).

A substituição de parte das resinas sintéticas pelo extrato tânico contribuiu para que o teor de sólidos diminuísse, uma vez que o teor de sólidos da solução de tanino era menor (próximo a $47 \%$ ).

Com relação ao tempo de formação de gel, não houve diferença significativa nos valores à medida que foi adicionado extrato tânico da casca de Pinus oocarpa ao adesivo UF. Esses resultados estão de acordo com os encontrados por Gonçalves (2000), estudando taninos de Mimosa caesalpiniaefolia; porém, diferentes dos encontrados por Gonçalves e Lelis (2009) e Tostes et al. (2004) em adesivos uréicos modificados com extratos tânicos de acácia-negra e eucalipto, respectivamente, onde os tempos de formação de gel observados foram diminuindo com a adição dos extratos tânicos.

Ao se adicionar solução de tanino de pinus ao adesivo fenólico, o tempo de formação de gel diminuiu consideravelmente, havendo diferença significativa. Isso explica a utilização, em alguns países, de taninos de pinus como endurecedores para adesivos FF, em razão de sua alta reatividade. Os baixos valores de tempo de gel dos extratos tânicos da casca de pinus evidenciam a grande reatividade dos taninos. O tempo de gel depende do $\mathrm{pH}$ e da estrutura da molécula de tanino, isto é, se os polifenóis são constituídos por unidades do tipo acácia (resorcinólicos) ou do tipo pinus (floroglucinólicos). O tipo resorcinólico é aquele que possui apenas uma hidroxila ligada ao carbono 7 no anel A, enquanto o tipo floroglucinólico possui hidroxilas nos carbonos 5 e 7. A reatividade do tanino depende do número de grupos hidroxílicos no anel benzólico A. Por isso, os diferentes tipos de taninos reagem diferentemente com formaldeído.

Cerne, Lavras, v. 20, n. 1, p. 47-54, jan./mar. 2014
O extrato tânico da casca de Pinus oocarpa apresentou um caráter ácido $(6,54)$. A adição de extrato aos adesivos UF e FF, promoveu a redução do $\mathrm{pH}$, em função desse caráter ácido do extrato tânico. $\mathrm{O}$ pH interfere tanto na reatividade, quanto na viscosidade das reações de tanino, sendo importante o controle dessa variável para impedir uma polimerização acelerada e consequente cura prematura do adesivo.

\section{CONCLUSÕES}

No processo de extração da casca de Pinus oocarpa, o tratamento com sulfito de sódio a $5 \%$ proporcionou o maior rendimento em taninos condensados, evidenciando a importância da utilização desse sal.

É possível utilizar tanino puro da casca de Pinus oocarpa como adesivo, em razão dos valores obtidos na viscosidade. Entretanto, o processo deve ser bem controlado, uma vez que o tanino mostrou-se bastante reativo.

A adição do extrato tânico da casca de Pinus oocarpa aos adesivos sintéticos contribuiu para aumento nos valores da viscosidade. Desta forma, a substituição de adesivos sintéticos por taninos da casca de Pinus oocarpa é possível somente até $10 \%$.

\section{REFERÊNCIAS}

AMERICAN SOCIETY FOR TESTING AND MATERIALS. ASTM D-1200: test method for viscosity by ford viscosity. Philadelphia, 1994.

CARNEIRO, A. C. O.; VITAL, B. R.; FREDERICO, P. G. U.; CARVALHO, A. M. M. L.; VIDAURRE, G. B. Propriedades de chapas de aglomerado fabricadas com adesivo tânico de angicovermelho (Anadenanthera peregrina) e uréia-formaldeído.

Revista Árvore, Viçosa, v. 33, n. 3, p. 521-531, maio/jun. 2009.

CARNEIRO, A. C. O.; VITAL, B. R.; PIMENTA, A. S.;

MORI, F. A. Reatividade dos taninos da casca de Eucalyptus grandis para produção de adesivos. Cerne, Lavras, v. 7, n. 1, p. 1-9, 2001.

COPPENS, H.; SANTANA, M. A. E.; PASTORE JUNIOR, F. Tannin-formaldehyde adhesive for exterior-grade plywood and particleboard manufacture. Forest Product Journal, Madison, v. 30, n. 4 , p. $38-42,1980$.

FERREIRA, E. S.; LELIS, R. C. C.; BRITO, E. O.; 
NASCIMENTO, A. M.; MAIA, J. L. S. Teores de taninos da casca de quatro espécies de pinus. Floresta e Ambiente, Seropédica, v. 16, n. 2, p. 30-39, 2009.

GONÇALVES, C. A. Utilização do tanino da madeira de Mimosa caesalpiniaefolia Bentham (Sabiá) como matéria prima alternativa para a produção de adesivos. 2000.78 p. Dissertação (Mestrado em Ciências Ambientais e Florestais) Universidade Federal Rural do Rio de Janeiro, Seropédica, 2000.

GONÇALVES, F. G.; LELIS, R. C. C. Propriedades de duas resinas sintéticas após adição de tanino modificado. Floresta e Ambiente, Seropédica, v. 16, n. 2, p. 1-7, 2009.

HILLIG, E.; HASELEIN, C. R.; SANTINI, E. J. Propriedades mecânicas de chapas aglomeradas estruturais fabricadas com madeiras de pinus, eucalipto e acácia-negra. Ciência Florestal, Santa Maria, v. 12, n. 1, p. 59-70, 2002.

HOINACKI, E.; MOREIRA, M. V.; KIEFER, C. G. Manual básico de processamento de couro. Porto Alegre: SENAI, 1994. $364 \mathrm{p}$.

HOONG, Y. B.; PARIDAH, M. T.; LOH, Y. F.; JALALUDDIN, H.; CHUAH, L. A. A new source of natural adhesive: Acacia mangium bark extracts co-polymerized with phenol-formadehyde (PF) for bonding Mempisang (Annonaceae spp.) veneers. International Journal of Adhesion and Adhesives, Guildford, v. 31, n. 3, p. 164-167, 2011.

LOPES, G. C.; NAKAMURA, C. V.; DIAS FILHO, B. P.; MELLO, J. C. P. Estudo físico-químico, químico e biológico de extrato das cascas de Stryphnodendron polyphyllum Mart. (Leguminosae). Revista Brasileira de Farmacognosia, São Paulo, v. 14, p. 24-27, 2003. Suplemento.

MELO, R. R. de; SANTINI, E. J.; HASELEIN, C. R.; STANGERLIN, D. M.; MULLER, M. T.; DEL MENEZZI, C. H. S. Avaliação das propriedades físico-mecânicas de painéis aglomerados de Eucalyptus grandis colados com ureiaformaldeído e tanino-formaldeído. Floresta, Curitiba, v. 40, n. 3, p. 497-506, jul./set. 2010.

MORI, C.; PIMENTA, A. S.; VITAL, B. R.; MORI, F. A. Uso de taninos de três espécies de Eucalyptus na produção de adesivos para colagem de madeira. Revista Árvore, Viçosa, v. 25, n. 1, p. 19-28, jan./fev. 2001.
MORI, F. A.; MORI, C. L. S. O.; MENDES, L. M.; SILVA, J. R. M.; MELO, V. M. Influência do sulfito e hidróxido de sódio na quantificação em taninos da casca de barbatimão (Stryphnodendron adstringens). Floresta e Ambiente, Seropédica, v. 10, n. 1, p. 86-92, 2003.

MORI, F. A.; VITAL, B. R.; LUCIA, R. M.; VALENTE, O. F.; PIMENTA, A. S. Utilização de resinas à base de taninos das cascas de Eucalyptus grandis w. Hill ex Maiden na produção de painéis compensados. Revista Árvore, Viçosa, v. 23, p. 455-461, 1999.

PAES, J. B.; DINIZ, C. E. F.; MARINHO, I. V.; LIMA, C. R. de. Avaliação do potencial tanífero de seis espécies florestais de ocorrência no semi-árido brasileiro. Cerne, Lavras, v. 12, n. 3, p. 232-238, jul./set. 2006.

PIZZI, A. Tannin-based adhesives. Journal of Macromolecular Science, Part C: Polymer Reviews, New York, v. 18, n. 2, p. 247-315, 1980.

PIZZI, A. Wood adhesives: chemistry and technology. New York: M. Dekker, 1983. 364 p.

PIZZI, A.; MITTAL, K. L. Handbook of adhesive technology. New York: M. Dekker, 1994. 680 p.

ROFFAEL, E. Über die Reaktivität von wäßrigen Rindenextrakten gegenüber Formaldehyd. Adhäsion, Cham, v. 20, n. 11, p. 306-311, 1976.

SANTANA, M. A. E.; PASTORE JUNIOR, F. Adesivos de tanino-formaldeído para aglomerados. Brasília: IBDF, 1981. 12 p. (Série Técnica, 2).

SILVA, R. V. Uso de taninos da casca de três espécies de eucalipto na produção de adesivos para madeira. 2001. 56 p. Dissertação (Mestrado em Ciência Florestal) - Universidade Federal de Viçosa, Viçosa, 2001.

TEODORO, Â. S. Utilização de adesivos à base de taninos na produção de painéis de madeira aglomerada e OSB. 2008. 91 p. Dissertação (Mestrado em Ciências Ambientais e Florestais) - Universidade Federal Rural do Rio de Janeiro, Seropédica, 2008.

TOSTES, A. S.; LELIS, R. C. C.; PEREIRA, K. R. M.; BRITO, E. O. Colagem de chapas de madeira aglomerada

Cerne, Lavras, v. 20, n. 1, p. 47-54, jan./mar. 2014 
com adesivo uréia-formaldeído (UF) modificado com tanino da casca de Eucalyptus pellita F. Muell. Floresta e Ambiente, Seropédica, v. 11, n. 2, p. 14-19, ago./dez. 2004.

VALENZUELA, J.; LEYSER, E.; PIZZI, A.;

WESTERMEYER, C.; GORRINI, B. Industrial production of pine tannin-bonded particleboard and MDF. European Journal of Wood and Wood Products, Berlin, v. 70, n. 5, p. 735-740, May 2012.

WISSING, A. The utilization of bark II: investigation of the Stiasny-reaction for the precipitation of polyphenols in Pine bark extractives. Svensk Papperstidning, Stockholm, v. 58, n. 20, p. 745-750, 1955.

Recebido: 6 de julho de 2011; aceito: 01 de julho de 2013.

Cerne, Lavras, v. 20, n. 1, p. 47-54, jan./mar. 2014 